Edith Cowan University

Research Online

ECU Publications 2011

2011

Evaluation of the Neurodegenerative Conditions Coordinated Care

Program (NCCCP) in Western Australia: Barriers to better service

provision

\author{
Susanne T. Bahn \\ Edith Cowan University \\ Margaret J. Giles \\ Edith Cowan University
}

Follow this and additional works at: https://ro.ecu.edu.au/ecuworks2011

Part of the Rehabilitation and Therapy Commons

10.1016/j.evalprogplan.2011.06.005

This is an Author's Accepted Manuscript of: Bahn, S. T., \& Giles, M. J. (2011). Evaluation of the Neurodegenerative

Conditions Coordinated Care Program (NCCCP) in Western Australia: Barriers to better service provision.

Evaluation and Program Planning, 35(1), 40-46. Available here

This Journal Article is posted at Research Online.

https://ro.ecu.edu.au/ecuworks2011/362 


\title{
Title: Evaluation of the Neurodegenerative Conditions Coordinated Care Program (NCCCP) in Western Australia: Barriers to better service provision
}

\begin{abstract}
This paper details a 2009-2010 evaluation of the Neurodegenerative Conditions Coordinated Care Program (NCCCP) commissioned by the Disability Services Commission (DSC) in Western Australia (WA). The program, run by the Multiple Sclerosis Society of WA (Inc.) provides in-home care and supports and respite for people who are under the age of sixty-five and diagnosed with a rapidly degenerative neurological condition. In 2009, the identified barriers to the better provision of services included: shortage of quality out-of-home respite providers, and convoluted paperwork requirements to qualify for the program. Some service providers were unaware of the program and so were not referring them into the NCCCP, a number of service providers were unwilling to refer their clients into the program, neurologists were unwilling to give a final diagnosis for a client, that would enable them to qualify for the service, there was a general lack of knowledge about what services were available in the general community, and existing equipment pools were under-resourced. In 2010, the study found that most of the issues had been addressed however, out-of-home respite services were still very limited especially in rural areas, and the eligibility criteria for entry to the program is confined to those 65 years and under. This paper discusses the issues that still remain with the program as examples of barriers to better provision of services.
\end{abstract}

Key Words: Rapidly degenerative conditions, in-home care, social program evaluation, barriers to better provision of services.

\section{Introduction}

The Disability Sector Health Check Committee was established by the Minister for Disability Services in August 2006 to examine the operations of the disability services sector in Western Australia. In the Interim Report on the Implementation of the Sector Health Check (Feb, 2008), Recommendation 20 proposed that the Commission examine alternative funding strategies for clients with fluctuating needs as a result of rapidly degenerating conditions (for example, Motor Neurone Disease, brain tumours, Huntington's Disease, and Batten's Disease). Pilot funding until June 2011 was allocated to The Multiple Sclerosis Society of Western Australia (Inc.) (MSS WA) through a competitive tender process to coordinate and deliver the Neurodegenerative Conditions Coordinated Care Program (NCCCP).

At this same time a formal evaluation of the program began, funded by the WA Disability Services Commission (DSC). The purpose of the NCCCP was to provide in-home services, equipment and respite care for people who are under the age of sixty-five and diagnosed with a rapidly degenerative neurological condition. The MSS WA (a not-for-profit organisation) provides service delivery in metropolitan and regional areas in WA (mostly in the south-west of WA due to the extensive size of the state), as well as acting, in a limited role since 2009, as a broker for a number of providers, for clients residing in regional areas (most clients reside within the metropolitan area). Table 1 shows the types of services the NCCCP provides.

Insert table 1 here

Prior to providing the NCCCP, MSS WA had only provided direct services to their specific clients most of whom had a diagnosis of multiple sclerosis. The purpose of the evaluation was in part to determine if they were the right 'fit' for delivery of the programme as well as to improve the programme service delivery as it progressed. 
Clients referred for NCCCP support (clients are referred by a specialist or through hospital clinics) under the program must be eligible for DSC services and fall within the specific program parameters. These parameters include the client being under the age of sixty-five at the time of referral and diagnosed with a neurological condition which results in a rapid decline in the individuals' capacity to independently carry out the activities of daily living as well as being likely to progress from the time of diagnosis to a requirement for full 24-hour care, or death, within a maximum of 3-4 years. The maximum expenditure per individual is $\$ 80,000$ per year or $\$ 10,000$ per month for two consecutive months. If a client exceeds this amount they may be exited from the program through sourcing alternative funding arrangements.

Each year in WA less than 200 people are likely to be affected by rapidly degenerative neurological disorders; however their needs are extensive. The average age of onset of these conditions is 50, with most clients dying within two years. They decline at a rapid rate with loss of ability to dress, feed and wash themselves; many are bedridden and require 24 hour care. On 28th February, 2009 the total number of clients registered for participation in the program was 120 . On $30^{\text {th }}$ June 201079 of the 136 registered clients were utilising services. Between January 2008 and 30 June 2010, 207 clients had accessed the NCCCP program.

Health care in Australia is funded by the federal government by the Medicare system. Additional services such as stay in a private hospital is paid for by the individual and usually supported by health insurance. The waiting lists for specialist referrals for neurodegenerative conditions are not extensive. Prior to the introduction of the NCCCP program, home care support services available to people who contracted a rapidly degenerative neurological condition were capped, inflexible and often required a coordinated approach by several providers. Many people with these conditions were admitted to hospital care in the final weeks or months of their lives.

The NCCCP provides a range of services including provision of loan equipment (particularly large pieces of equipment, such as hoists which were either those purchased by the program or hired); training in equipment use; transport of equipment to homes in the metropolitan and rural areas; respite (both in-home and out-of-home); domestic assistance; help with personal care (such as showering, dressing); access to allied health professionals for assessment (such as Occupational Therapists); counselling; massage services; advice on funeral and financial arrangements; and limited short-term support for the carer once the client is deceased. The aim of the program is to provide in-home care for as long as possible to allow for the client to live and die in their own home rather than in a clinical hospital setting wherever possible. Earlier research by Giles and Lewin (2008) into the needs of adults with neurodegenerative disorders concluded that, for most participants in their study, continuing to live in the community was their primary goal. 


\section{Rapidly degenerative conditions and the support they need}

The types of home care support services available to people with rapidly degenerative conditions in the last weeks and months of their lives have, in the past, been hospital or hospice based or a multiplicity of services that are informally packaged, albeit insufficient and inflexible, to provide care. When people are in rapid decline they require one-on-one continual care. This is costly for the community as valuable hospital beds are taken up for as long as two years before the person dies. Additionally, having a family member hospitalised for such a period of time places an emotional and logistical strain on the family. The provision of the NCCCP allows the client continue to live in the community and, perhaps, die at home as is often the preference by the patients and their families. This is beneficial for the client, the family and the community and has in some instances lengthened the life of some with rapidly degenerative conditions (Cockram, Bahn, Giles \& Cooper, 2010).

A number of Australian and overseas studies have been conducted on unmet home care support needs for people with degenerative neurological diseases. Studies indicating the need for multidisciplinary care include:

Kristjanson (2003; 2004) who interviewed people with Multiple Sclerosis, Motor Neurone Disease, Parkinson's Disease and Huntington's Disease, their carers and health care professionals in a two part study of the effectiveness of Australian models of palliative care for people with these disorders. Kristjanson (2004) reported that people with neurodegenerative disorders and their carers preferred service provision within the home, but that care in the home required a plethora of services including care workers, allied health professionals, general practitioners (GPs) and specialists, respite and palliative care.

Giles and Lewin (2008a; 2008b) investigated and quantified the gaps in home support for people with Multiple Sclerosis, Motor Neurone Disease, Parkinson's Disease and Huntington's Disease in Western Australia. Their findings included the recognition of a need for improved home care support that is individualised, case managed, flexible and timely.

The WA Department of Health (2008, p.7), who prepared a report as a framework for care, on the Motor Neurone Disease services for Western Australia recommended that coordinated multidisciplinary care for patients with Motor Neurone Disease was essential at all levels of health care. They maintained that "a planned and well managed disease course based on the needs and wishes of the person with Motor Neurone Disease assists in improving quality of life. This can also prolong survival whilst being cost effective in reducing unplanned and possibly unnecessary hospitalisations”.

Skirton and Glendinning's (1997) study in the UK and McGarva's (2001) study in Scotland with Huntington's Disease patients argued for multi-disciplinary assessment for people with degenerative diseases and support as well as practical collaboration between agencies to support their carers. van Teijlingen, Friend and Kamal's (2001, p.397) study in Scotland with Motor Neurone Disease patients found that "the formal health and social care sectors in conjunction with voluntary organisations are only partially managing the trajectory of patients with a rare progressive degenerative disease”. In the US, Habbermann and LindseyDavis (2005) found that carers need information (and training) not only about how to care, but also about how to care for themselves. 
Evaluations such as that conducted in Australia by Dawson, Kristjanson, Toye and Flett (2004) who interviewed people with Huntington's disease and their carers found that the service needed to provide flexibility in their provision of practical and psychological support. Pozzilli, Brunetti, Amicosante, Gasperini, Ristori, Palmisano, et al. (2002, p.250) interviewed MS patients in Italy to compare, the cost effectiveness of home based care versus hospital care found that "comprehensive planning of home based intervention implemented by an interdisciplinary team and designed specifically for people with multiple sclerosis may provide a cost-effective approach to management and improve the quality of life”.

However, Tribe, Longley, Fulcher, Faine, Blagus, Pearce, et al. (2006) in their study of Multiple Sclerosis patients living in New South Wales, Australia, found that for those living further away from major cities personal support needs were less reliable.

In summary, these Australian and international studies, all of which interviewed or surveyed patients with neurodegenerative conditions, carers and/or health professionals, found unmet needs for home care support and recommended a multi-faceted, case management approach. These in-home services improve the quality of life for the patient and can also prolong life expectancy whilst also reducing unplanned or unnecessary hospitalisations. The NCCCP program has gone to considerable lengths to address these issues.

\section{Methodology}

An interpretative phenomenological analysis (IPA) (Smith, 1996) was adopted in this study to analyse the data collected. This methodology acknowledges that the relationship between what people say and what they think and feel is complex. Accordingly, IPA adopts an interpretative approach to analysis which acknowledges that the research process is a dynamic one. The depiction of participants' personal worlds involves two stages of interpretation: participants attempt to make sense of their experiences, and the researchers attempt to make sense of, and interpret, the participants making sense of their experiences.

While acknowledging that one cannot obtain direct access to the participants' worlds, the role of the researcher is to engage with participants' accounts in such a way that an "insider perspective" is obtained. However, in doing so, it is recognised that a researcher's interpretation is influenced by that researcher's own thinking and understanding. Rather than being viewed as biasing, these factors are necessary in order to be able to interpret and make sense of someone else's experiences. For example their thoughts and feelings, the IPA researcher's role is to interpret their mental and emotional states from what they do and say. Such inferences are made with recognition of the participant's contextual and cultural background.

IPA studies are conducted on homogeneous small samples. The aim of such studies is to provide an in-depth analysis of the perceptions and understandings of the selected group, as opposed to making generalised assertions about larger populations. As such, samples for IPA studies are purposively drawn, and utilise an idiographic method of inquiry. The key research question for this study was: What are the barriers for better provision of services for people with rapidly degenerating neurological conditions? In this study the results were fed back to the participants in the form of a final report (Cockram et al. 2010) in an iterative process as per an IPA methodology. 


\section{Sample}

The sample was purposively selected for the research project and included representatives of service providers and clients and carers using the service from both city and rural areas. In some instances carers whose partner/child had died were interviewed to collect information that followed the service delivery until it was no longer required by the client.

Because the clients in this research study are nearing the end of their illness and consequently the end of their lives, the sample of clients interviewed had to be flexible. It was anticipated within the research design that it would not be possible to follow all the selected clients throughout all three stages of the evaluation. Where possible however, the carer of the deceased client subsequently contributed to the data collection.

Three sets of data were collected for the project: qualitative data collected in 2009 (stage 1) and 2010 (stage 2); and quantitative client and service data for the period January 2008 to June 2010. The researchers did not interview people who were not registered into the program who had rapidly degenerative conditions.

Qualitative data was collected through semi-structured face-to-face interviews, and telephone interviews. Semi-structured face-to-face interviews were conducted in most cases. However, where this was not possible due to distance, availability of participants and stated participant preference, telephone interviews were substituted. The interviews and were held for the duration of 15-90 minutes. A full ethics clearance was obtained prior to the interviews and counselling services were offered to both participants and the researchers for those who felt they needed to access them. These were particularly vulnerable participants discussing a very sensitive topic. For the stage one data collection three representatives from MSS WA, six representatives from service providers, five clients and eleven carers were interviewed. A total of twenty-five interviews were conducted. Because of failing health of clients, in three instances the carer and the client were interviewed together. Twelve interviews were conducted in face-to-face mode, and thirteen were conducted as telephone interviews.

For the stage two data collection three representatives from MSS WA, ten representatives from service providers (including two referring neurologists, three Motor Neurone Disease Association of WA representatives, representatives from the Neurological Council in Bunbury (regional centre of WA) and the WA Health Department Neurosciences Unit, and one representative from the Creutzfeldt-Jakob Disease Association), eight clients and eleven carers were interviewed. The interviews with the representatives from MSS WA were faceto-face and the interviews with the service providers were all conducted by telephone. The client and carer interviews were predominantly telephone interviews with one face-to-face. Two telephone interviews were conducted as joint carer/client interviews. Three of these participants were interviewed in the stage one data collection. Two of the carers interviewed had clients that had recently deceased.

The quantitative data were analysed to provide a summary of per client costs at 30 June 2010 as well as disaggregated average costs per type of service, and to provide an estimate of the average and total cost of the program for clients with Motor Neurone Disease in 2010 and a projection of the average and total cost of the program for this client group in 2021. This data was used by DSC to provide detail in their business case to secure ongoing funding. 


\section{Participants on the program}

On 28th February, 2009 the total number of clients registered for participation in the program was 120 . On $30^{\text {th }}$ June 2010 there were 79 clients utilising services from 136 registered clients. Clients who registered but did not access services were those who were in the early diagnosis of their condition. The NCCCP encourages early detection and registration so that in the event their condition begins to rapidly progress services can be ramped up quickly. Between January 2008 and 30 June 2010, 207 clients had accessed the program. There were fifty-nine male clients residing in the metropolitan area and sixteen rural residents in 2009 compared to 76 male clients in the metropolitan area and 5 rural residents in 2010. There were thirty-six female clients residing in metropolitan residences and nine in rural areas in 2009 compared to 50 female clients in the metropolitan area and 5 rural residents in 2010.

Table 2 indicates the number of male clients and their age range and Table 3 indicates the number of female clients and their age range. In 2009, $62 \%$ of the clients were male; $38 \%$ were female. In 2010, $60 \%$ of the clients were male; $40 \%$ were female. Eight children under the age of 10 years, or $6 \%$ of the total were registered in the program in 2009. By June 2010, two of these children had deceased with four of the remaining six children residing in regional areas. The inclusion of children in the program was not anticipated by the service provider. The median age group for client involvement is fifty-one years and over with seventy-two clients registered (60\%) in 2009 and ninety-three clients (68\%) in 2010.

\section{Insert tables 2 and 3 here}

Table 4 depicts the types of conditions and the number of clients with these conditions serviced by the program as at $28^{\text {th }}$ February 2009 and at $30^{\text {th }}$ June 2010.

\section{Insert table 4 here}

Over the period January 2008 to June 2010, 207 clients were provided with 16,376 services under the NCCCP. Most NCCCP clients received services of assessment (111 clients) and/or case management (168 clients). The most popular types of care services were respite (98 services per client) and personal care (84 services per client). On average clients received 76 services each with an average cost per client of $\$ 10,205$. The total cost of the program under the types of services listed in Table 9 was \$2,194,169 over this period. For clients with MND ( $n=104$ ), who make up $54 \%$ of all NCCCP clients, the total cost of the program was about $\$ 469,000$ p.a. as shown in Table 5.

Insert table 5 here

\section{Results and Discussion}

It was apparent that the average cost of the NCCCP program for individual clients masked differences between clients over a range of characteristics. A simple model of the relative contribution of these characteristics to average cost is shown in Table 6. The average cost of servicing NCCCP clients was found to be less expensive for clients living in the metropolitan area compared with those in regional areas; much more expensive for clients with MND compared with clients with other disorders (coefficient is statistically significant at the $1 \%$ level); less expensive for male clients compared with female clients ; and decreases as client age increases, that is, servicing older clients is cheaper than servicing younger clients (coefficient is statistically significant at the $10 \%$ level). 


\section{Insert table 6 here}

The explanatory power of the model summarised in Table 6 is very weak - less than $5 \%$ of the variation in average cost is explained by the regressors in the model. This is not unexpected as the information in the client database, compiled for the purposes of managing NCCCP clients, does not include information about characteristics of the clients that may be contributing to the variation in cost, such as severity of the disorder, level of mobility and functioning, type of housing, and family, other than carer, support.

\section{Insert table 7 here}

Table 7 shows that NCCCP clients that were current clients (still in the program) at 30 June 2010 used more hours on average at a higher average cost than clients who had already exited the program due to their death or other reasons (no longer eligible, etc). This is not unexpected as many clients who entered the program in its first year were already in need of considerable support but only for a very short period. Clients who entered the program later have experienced support ranging from very little services and no equipment to an increasing requirement for services and equipment as their conditions deteriorate. Clients who exited the program by moving into a nursing home or to an area not serviced by the NCCCP program, or who became ineligible or were referred to other more appropriate services had, as expected, shorter duration in the program.

In 2009, the NCCCP provided a range of services including training in equipment use; transport of equipment to homes in the metropolitan and rural areas; respite (both in-home and out-of-home); domestic assistance; help with personal care (such as showering, dressing); access to health professionals for assessment (such as Occupational Therapists); advice on funeral and financial arrangements; and limited short-term support for the carer, up to 3 months, once the client had deceased. The services remained largely the same in 2010 except that counselling and massage services were included as well as additional on-going access to out-of-home respite, in a homely setting, every three months to clients who needed it and was particularly helpful to clients who did not reside in the metropolitan area. The need for a large range of services for people with rapidly degenerative neurological conditions was also identified by Dawson et al (2004), Pozzilli et al (2002), Skirton and Glendinning (1997), and McGarva (2001).

The NCCCP program also provided loan equipment. The most common items of equipment were bed/chair raisers $(n=74)$, electric beds $(n=17)$, bedside tables $(n=16)$, threshold ramps $(n=16)$, manual wheelchairs $(n=15)$, raised over toilet seat with arms $(n=14)$ and MP3 players $(n=10)$. Other items of equipment were used as aids for specific needs by some clients. The initial purchases of equipment in 2008 were subsequently supplemented in 2009 and 2010 for some popular items such as chair raisers. Of the 204 clients in the NCCCP program from 2008 to 2010 inclusive, 87 used at least one item of equipment. The maximum number of items on loan to any one client was 11, although some of these items were only being trialled and thus returned quickly. 
Most equipment was loaned to clients until they exited the program (due to death or client transfer to hospital or residential care). Some equipment was loaned to clients to trial its efficacy in relation to their needs. Other aids or equipment were specific to certain levels of functioning and hence were used in the short term before replacement with other equipment. For example, manual wheelchairs would be on short term loan whilst the clients had use of their hands and arms; they could swap these manual chairs for electric chairs as their upper body strength deteriorated. Returned equipment was made available to existing clients including clients new to the program.

Most equipment was bought for the program. However, some equipment was hired for the program from other services or suppliers. A few pieces of equipment were found to be faulty or became damaged but it is unclear from the equipment records whether these items were replaced, replaced without cost or replaced with a new purchase by the NCCCP program. Donations of equipment are also used in the NCCCP program.

The program had two significant operational changes since September 2009; one being a change of coordinator and the other being the use of MSS WA employed in-home care workers in lieu of brokered agency staff. In 2010, the program employed forty part-time care support worker staff members to provide personal care instead of relying on outside agency staff to perform these duties as in the previous year. This resulted in agency staff being required only for clients outside the metropolitan area; accounting for only $10 \%$ of those on the program. Clients have reported that the change to staffing provided continuity in the staff providing personal care rather than the large staff turnover from agency staff in the past. Clients appreciated having the same person/s attending to their needs as the staff got to know their specific requirements and they were not faced with explaining to new staff how to care for them.

The program evaluation indicated that overall the clients are happy with the service, particularly in that the services were available at no financial cost to them. There is considerable support for the provision of in-home care by NCCCP staff compared to services delivered by other service provider agency staff. The data revealed that in general most service providers, carers, clients, and the MSS WA were satisfied with their ability to deliver the services in the future. However, for this paper, rather than only reporting in detail the positive findings of the evaluation; instead the article discusses the remaining issues residing with the program as a guide for the better provision of in-home and out-of-home services for people with rapidly degenerative conditions.

Most of the operational issues identified in the first year of the program were addressed in the program's second year. The willingness of MSS WA to embrace the program requirements and make the necessary changes is a credit to them. Nonetheless some issues, generally outside the control of the program provider, remain unresolved. These issues - accessing services in rural areas, the program eligibility criteria, and effective service provision for children and young people with rapidly degenerative conditions - are discussed below and are supported by verbatim quotes obtained in the interview data collections in stages one and two. The quotes that appear have been selected as the example that most cogently expresses the ideas relevant to the themes that emerged in the study.

\section{Accessing services in rural areas}

The program is efficient in providing equipment to clients living in country areas however, supplying in-home care and respite remains problematic. Tribe et al. (2006) also found that services for rural clients were less reliable. The program is reliant on brokering other service 
providers to perform in-home services and clients are required to travel to the metropolitan area for out-of-home respite.

I guess the biggest challenge that I saw was the fact that we are based in regional, rural and remote and it is something I recognize on a daily basis. In this case it was difficult to just work out which funding we needed to get people or workers from Perth or qualified staff because we could not actually identify any trained paediatric palliative staff in the region and that was a great gap in all honesty.

There needs to be a systemic better management of delivery of equipment to people. It should be regional, perhaps in the metro area it's aligned with the health region but also include the country because there needs to be money and infrastructure to manage equipment pools to make sure the equipment is cleaned and maintained and also tracked.

The difficulty for many in-home programs to provide adequate services in regional areas is not new. This is particularly evident in Western Australia as it is a large state with an area of 2,525,500 km2 and a population of 1.8 million; $80 \%$ of which live in the metropolitan area. Approximately 400,000 people reside in country areas in WA (ABS, 2010). The large distances between people with degenerative diseases in rural WA have meant that the program relies on agency staff to deliver services; approximately $10 \%$ of the services are delivered in this way. There is no easy solution for this issue.

\section{Eligibility criteria}

The NCCCP has strict eligibility criteria that the Program Coordinator adhered to on most occasions. However, when they were unsure of eligibility they referred the case to a specialist consultant neurologist who assisted in defining whether they met the diagnostic criteria. Additional support or approval was sought from time to time from DSC. In some instances if a client was not quite eligible for the NCCCP they approved three months service and reassessed them for future funding. Eligibility restrictions such as requiring clients to be under 65 years of age and to die, or enter 24 hour care, within a four year period have had some clients ineligible for the program or they had difficulties in receiving continued services after the 4 year NCCCP service provision. When presented with people who were ineligible the MSS WA endeavoured to direct them to other services provided by the WA Department of Health.

I think though that sometimes the eligibility has been a bit exclusive. Because it is saying that you want people to be dead within a timeframe or to have gone into 24hour care. There are a lot of folks that we can't help through the program based on their age and their situation even though we would really like to.

Originally we were refused the funding and it was basically to do with criteria but I didn't take it lying down I fought for it because I saw the need for it and I wasn't going to stop because I thought that the client needed the service. If it wasn't for somebody like myself, who was prepared to work for it, this lady could have been in a nursing home. It had strong implications if we just stuck by the current criteria.

There are some who've had their diagnosis longer than the limitation so we've had a few of those. You know people can survive anywhere from six months to 20 years but mostly it's about two to three years. We haven't got time to mess around trying to 
decide where people fit in the program; you need to treat it and service now because it can be pretty rapid for people.

The issue that remains for the program is that the level of service for those accessing the NCCCP prior to reaching age 65 is more comprehensive and better meets the needs than traditional aged care services in WA. The dilemma for the program was how to best support those transitioning from the NCCCP to aged care. MSS WA approached the DSC on a caseby-case basis to continue to provide support on the program for those that were in this position. It could be argued that services for people with degenerative conditions require this type of flexibility and compassion.

There are some people who are referred in and part of the exit criteria for this grant is that if we take someone in who is eligible but then they plateau and it looks like that they are going to continue living for an extended period of time what we agreed was that we would do a Care Aged Package (CAP) application on their behalf that we wouldn't pull the services out until they got funded. So what we have identified is that there are some people who have been referred to us who are stretching the boundaries a little. So we have taken them on a temporary basis and then complete their CAP applications on their behalf.

In developing the age criteria for the program it was not envisaged that children would become clients of the service. In the first year of the program, issues with the care needs of children on the program were identified. The MSS WA appealed to the DSC to provide better flexibility for children on the program and this issue was resolved in 2010 with four children receiving services. It could be argued that flexibility in criteria including age parameters and the types of services delivered is required by programs providing in-home care to ensure a better provision of services.

\section{To summarise}

In 2009, the identified barriers to the better provision of services within the NCCCP included: a shortage of quality out-of-home respite providers, and convoluted paperwork requirements to qualify for the program and services within the program. Some service providers were unaware of the program and were not referring them into the NCCCP, a number of service providers were unwilling to refer their clients into the program as they feared they would lose them as their clients, neurologists were unwilling to give a final diagnosis for a client that would enable them to qualify for the service, there was a general lack of knowledge about what services were available in the community, and equipment pools were under-resourced.

In 2010, the review found that many of these barriers have been overcome. MSS WA achieved this by increasing in-home respite services; however out-of-home respite services remain very limited especially in rural areas (Tribe et al., 2006). In addition MSS WA communicated more with other service providers to increase the knowledge of the program which facilitated an increase in referring more clients into the program and increased self referrals. The service providers streamlined the registration paperwork and now only refer those they believe will meet the requirements.

Service providers indicated that the program filled significant gaps in other care packages as programs such as the Home and Community Care (HACC) and Extended Age Care at Home $(\mathrm{EACH})$ programs had age criteria and restricted hours that did not cater to the needs of people with rapidly degenerative conditions. However, service providers, carers and clients 
have all reported that they remain unclear as to what they can access, when they can access the services and how much they can have. The participants in the program have been made more aware of the services that the program can provide with additional brochures and facts sheets issued to them. Equipment pools are better resourced and maintained and more appropriate equipment to better meet the needs of clients has been purchased, for example: slimmer electric wheelchairs with reclining backs.

A continuing perceived issue from most respondents to better provision of services was the need for more funding and continued funding, accessing services in rural areas and the eligibility criteria. MSS WA together with DSC has lobbied the federal and state governments for continued and increased funding based on the evaluation. The addition of children on the program and the specialised care they require was identified in 2009 and addressed.

In 2010, issues such as accessing services in rural areas still remained. Although, the program is efficient in providing equipment to clients living in country areas however, supplying inhome care and respite remains difficult. The NCCCP program is reliant on brokering other service providers to perform in-home services and clients are required to travel to the metropolitan area for respite.

\section{Conclusion}

Overall, the clients and their families are happy with the service, particularly because services were available at no financial cost to them. There is considerable support for the provision of in-home care by NCCCP staff compared to services delivered by other service provider agency staff. Clients are provided with extensive services, equipment and in-home care to make their last days more comfortable. Additionally respite is provided for other family members who undertake additional caring roles for the clients. The program has been successful in addressing a previously not catered for need.

Generally, the service providers that have been involved in the NCCCP reported that they did not perceive any gaps in the program. The funding is additional to original services and allows service providers the flexibility to address their client's individual requirements. The only gaps emerging from the data were challenges with the quality out-of-home respite, generally in hospice settings, and the limited choice of providers and the difficulty in accessing care workers in rural and remote areas; a systemic issue. These are not faults with the program; rather they are gaps in community services and practical and economic difficulties with provision of social and community services in rural and remote locations in Western Australia.

However, the eligibility criterion remains a constraint for the program. The cut off age for registering for the NCCCP of 'under 65 years' is viewed by most providers as problematic with those individuals accessing support through aged care programs that may not be as comprehensive as the NCCCP in providing the care older individuals with rapidly degenerative diseases require. There is a genuine concern for equity. Improving aged care funding to include this type of service would provide equity for those currently ineligible for the program. The provision of quality care through the program has in some cases prolonged the life of some clients with neurodegenerative diseases so that they have not died within the four years as specified in the eligibility criteria. Although these events are to be celebrated as a program success, they also pose a problem for the program in that these clients have used their allotted hours and the staff as well as carers and clients have had to become creative in 
accessing other funding to top up the NCCCP hours to continue the required level of support. To maintain the service provision of the NCCCP to 2021, assuming the MND client growth rate across all client groups, would require an increase in funding of about $157 \%$. This increase reflects both the increasing cost of health service provision and also the growth in the population of eligible clients.

\section{Acknowledgements}

The author would like to thank the staff at the Multiple Sclerosis Society of WA (Inc) for their assistance with this project and the Disability Services Commission for the funding; with specific thanks to Richard Matthews and Nick Cantatore.

\section{References}

Australian Bureau of Statistics (ABS) (March, 2010). Australian Demographic Statistics, March 2010, Available at: http://www.abs.gov.au/ausstats/abs@.nsf/mf/3101.0

Cockram, J., Bahn, S., Giles, M. \& Cooper, T. (2010). Evaluation of the Neurodegenerative Conditions Coordinated Care Program (NCCCP) Final Report, Edith Cowan University.

Dawson, S., Kristjanson, L., Toye, C., \& Flett, P. (2004). Living with Huntington's Disease: Need for supportive care. Nursing and Health Sciences, 6, 123 - 130.

Department of Health, Western Australia (2008). Motor Neurone Disease Services for Western Australia. Perth: Health Networks Branch, Department of Health, Western Australia.

Disability Services Sector Health Check (February, 2008). Interim report on the implementation of Sector Health Check report recommendations, Available at: http://www.disability.wa.gov.au/dscwr/_assets/main/report/documents/doc/shcimplementatio $\underline{\mathrm{n}}$

Giles, M. \& Lewin, G. (2008a). An investigation into the home support needs of adults living with Multiple Sclerosis, Huntington's, Parkinson's and Motor Neurone Diseases: Client and carer interviews. Osborne Park, Silver Chain. Available at: http://www.silverchain.org.au/assets/files/NDP-Report-2-Client-and-Carer-Interviews.pdf

Giles, M. \& Lewin, G. (2008b). An investigation into the home support needs of adults living with Multiple Sclerosis, Huntington's, Parkinson's and Motor Neurone Diseases: Key issues and unmet needs - health, allied health and service provider perspectives. Osborne Park, Silver Chain. Available at: http://www.silverchain.org.au/assets/files/NDP-Report-5Provider-Key-Issues.pdf

Habbermann, B., \& Lindsey-Davis, L. (2005). Caring for Family with Alzheimer's Disease and Parkinson's Disease: Needs, Challenges and Satisfactions. Journal of Gerontological Nursing, 31(6), 49 - 54.

Kristjanson, L. (2003). Assessing the Extent to which Existing Models of Supportive Care Service Delivery meet the Needs of People living with Multiple Sclerosis, Motor Neurone, Parkinson's and Huntington's Diseases and their Families. Perth, Western Australia: Edith Cowan University. 
Kristjanson, L. (2004). Assessment of the Effectiveness of Australian Models of Palliative Care Delivery in Four Neurodegenerative Disorders (Final report). Perth: Edith Cowan University.

McGarva, K. (2001). Huntington's Disease: Seldom Seen - Seldom Heard? Health Bulletin, 59(5).

Pozzilli, C., Brunetti, M., Amicosante, A., Gasperini, C., Ristori, G., Palmisano, L., et al. (2002). Home Based Management in Multiple Sclerosis: Results of a Randomised Controlled Trial. Journal of Neurology, Neurosurgery and Psychiatry, 73, 250 - 255.

Skirton, H., \& Glendinning, N. (1997). Using Research to Develop Care for Patients with Huntington's Disease. British Journal of Nursing, 6(2), 83 - 90.

Smith, J.A. (1996). Beyond the divide between cognition and discourse: using interpretative phenomenological analysis in health psychology. Psychology \& Health, 11, 261-271.

Tribe, K., Longley, W., Fulcher, G., Faine, R., Blagus, L., Pearce, G., et al. (2006). Living with Multiple Sclerosis in New South Wales, Australia, at the Beginning of the $21^{\text {st }}$ Century: Impact of Mobility Disability. International Journal of MS Care, 8(1), 19 - 30.

van Teijlingen, E., Friend, E., \& Kamal, A. (2001). Service Use and Needs of People with Motor Neurone Disease and Their Carers in Scotland. Health and Social Care in the Community, 9(6), 397 - 403. 